\title{
1 Bilateral differences in hamstring coordination in previously injured elite
}

13

14

15

16

17

18

19

20

21

22

23

24

25

26

27
2

3

4

5

$6{ }^{1}$ French Institute of Sport (INSEP), Research Department, Laboratory Sport, Expertise and

7 Performance (EA 7370) Paris, France

$8{ }^{2}$ University of Nantes, Faculty of Sport Sciences, Laboratory Movement, Interactions,

$9 \quad$ Performance (EA 4334), Nantes, France

$10{ }^{3}$ Institut Universitaire de France (IUF), Paris, France

$11{ }^{4}$ The University of Queensland, School of Health and Rehabilitation Sciences, Brisbane,

\section{athletes}

\author{
Simon AVRILLON ${ }^{1}$, François HUG $^{2,3,4}$, Gaël GUILHEM ${ }^{1 *}$
}

Australia

\section{"Correspondence and reprints:}

Gaël Guilhem, PhD

Institut National du Sport, de l'Expertise et de la Performance

Département de la Recherche

Laboratoire Sport, Expertise et Performance (EA 7370)

11, avenue du Tremblay

75012 Paris

France

Tel: +33 (0)1 41744336

Fax: $+33(0) 141754535$

e-mail: gael.guilhem@insep.fr

Running title: Injury-induced changes in muscle coordination 


\section{ABSTRACT (250 words)}

29 Background: Hamstring strain injuries (HSI) involve tissue disruption and pain, which can

1 trigger long-term adaptations of muscle coordination. However, little is known about the effect of previous HSI on muscle coordination, and in particular, after the completion of rehabilitation and in the absence of symptoms. This study aimed to determine if elite athletes with a prior unilateral HSI have bilateral differences in coordination between the hamstring muscle heads after returning to sport.

Methods: Seventeen athletes with a unilateral history of biceps femoris injury participated in the experiment. Surface electromyography (EMG) was recorded from three hamstring muscles (biceps femoris $[\mathrm{BF}]$, semimembranosus [SM], and semitendinosus [ST]) during submaximal isometric torque-matched tasks. The product of normalized electromyographic amplitude with functional cross-sectional area (PCSA) and moment arm was considered as an index of muscle torque for each of the three hamstring heads.

Results: The contribution of the injured muscle to total knee flexor torque was lower compared to the uninjured limb $(-10.8 \pm 27.5 \% ; P=0.038)$. This reduced contribution of $\mathrm{BF}$ was compensated by a higher contribution of the SM muscle in the injured limb $(+17.2 \pm 27.4 \% ; P=0.007)$. These changes resulted from a decreased contribution of PCSA from the injured muscle $(\mathrm{BF})$, and an increased contribution of activation from an uninjured synergist muscle (SM).

Conclusions: Bilateral differences in coordination were observed in previously injured athletes despite the completion of rehabilitation. Whether these bilateral differences in hamstring coordination could constitute an intrinsic risk factor that contributes to the high rate of hamstring injury recurrence remains to be investigated. 


\section{NEW \& NOTEWORTHY:}

54 We used an experimental approach combining the assessment of muscle activation, 55 physiological-cross sectional area and moment arm to estimate force-sharing strategies among 56 hamstring muscles during isometric knee flexions in atheletes with an history of hamstring 57 injury. We observed a lower contribution of the injured biceps femoris to the total kne flexor 58 torque in the injured limb than in the contralateral limb. This decreased contribution was 59 mainly due to a selective atrophy of the injured biceps femoris muscle and was compensated 60 by an increased activation of the semimembranosus muscle.

61

62 KEYWORDS

63 Hamstring injury; Torque-sharing strategies; Atrophy; Muscle activation; Muscle 64 coordination 
65

66

67

68

69

70

71

72

73

74

75

76

77

78

79

80

81

82

83

84

85

86

87

88

89

\section{INTRODUCTION}

A hamstring strain injury is a leading cause of unavailability for training and competition in numerous sports (27). Due to their high incidence and reinjury rate (29), prevention is a main challenge for both coaches and clinicians. Most of these strain injuries involve tissue disruption in the biceps femoris (BF) muscle (11). These lesions are associated with pain and functional losses due to mechanical alterations. In addition, changes in muscle activation and neuromuscular inhibition may occur $(13,34)$.

Some theories propose that movement is modified in the presence of pain in order to unload the painful/injured tissue $(20,26)$. Although unloading the injured muscle seems logical during the acute phase of hamstring strain injury (34), it is unclear whether this adaptation persists after rehabilitation when pain has resolved. It is important to address this question as previous work suggested that altered coordination strategies might have an immediate benefit for the system, but that the persistence of these changes might have negative long-term consequences and increase reinjury risk $(10,20)$.

Muscle coordination relates to the distribution of force among individual muscles to produce a given motor task (22). As such, the study of muscle coordination requires the consideration of individual muscle force rather than muscle activation alone (22), especially within the context of muscle injury where both muscle activation and muscle force-generating capacity are likely to be altered. During isometric contractions, individual muscle force can be estimated from information on both activation and physiological cross-section area (PCSA). This approach considers that a difference in force-generating capacity between synergist muscles is mainly attributable to their difference in PCSA. This is reasonable when considering submaximal isometric knee flexions during which neither the force-length relationship nor the specific tension is expected to vary greatly between the hamstring muscle heads, because of their similar action on both the knee and hip joints (39) and their similar 
90 fiber-type content (14). Using this approach, Avrillon et al. (2) reported large interindividual

91 variability in muscle coordination strategies between the hamstring muscles, which is in some

92 cases detrimental for motor performance (i.e., the higher the activation variability, the lower

93 the time to exhaustion).

94 Although previous studies reported an alteration in either muscle force-generating capacity or 95 activation after hamstring strain injury, none of the studies considered these parameters 96 together, making it complicated to infer changes in muscle coordination. Silder et al. (37) 97 reported a selective decrease in volume of the injured muscle (BF in most of the participants) 98 six months after injury. Although this result might suggest a reduced contribution of this 99 injured muscle to joint torque, muscle activation was not assessed. Schuermans et al. $(35,36)$ reported a larger contribution of BF and SM muscles compared to ST up to two years after an

101 injury, but did not consider muscle volume or PCSA. In addition, the fragmented information 102 in these studies regarding injury localization made interpretation of the observed adaptations 103 difficult.

104 Here, we assessed muscle coordination in elite athletes with a hamstring injury in the previous 105 seven months that have returned to sport. We tested the hypothesis that the contribution of the injured muscle to submaximal knee flexion tasks will be reduced in the injured limb

107 compared to the non-injured limb. This reduced contribution would be a combination of both 108 a smaller volume and a lower activation of the injured muscle compared to uninjured muscles.

\section{METHODS}

\section{2.1. $\quad \underline{\text { Participants }}$}

112 Seventeen elite male sprinters and long jumpers volunteered for the study (age: $26.3 \pm 5.5 \mathrm{yr}$, 113 height: $1.79 \pm 0.05 \mathrm{~m}$, body mass: $74.4 \pm 8.1 \mathrm{~kg}$ ). They had a history of injury to the biceps 114 femoris long head (BFlh). Note that the semitendinosus (ST) was also involved in the injuries 
115 of three athletes (Table 1). All athletes were free from lower limb pain at the date of the

116 experiment and were able to perform maximal knee flexions. All participants were informed

117 regarding the nature, aims and risks associated with the experiments before they gave their

118 written consent to participate. Experimental procedures were approved by the local ethical

119 committee (reference no. 3418, RCB no. 2016-A00715-46) and conformed to the Declaration 120 of Helsinki.

121

\section{$122 \quad$ 2.2. Injury history}

123 All participants had a unilateral strain injury of the BFlh within the past seven months (Table

124 1). The average delay between injury occurrence and testing was $98.2 \pm 53.3$ days (range 22-

125198 days). We defined a hamstring injury as an acute pain in the posterior thigh that occurred

126 during a sprint and resulted in the immediate termination of the training session or

127 competition. Each injured athlete underwent an MRI $(n=9)$ or an ultrasound $(n=8)$ exam

128 performed by a radiologist within the week following injury. Athletes met inclusion criteria

129 when the precise localization and the grade of the injury was confirmed by the exam. Their

130 injuries caused training activities to stop for 32.5 \pm 17.5 days (range 14-70 days). All athletes

131 completed a supervised rehabilitation protocol provided by a qualified physiotherapist. In the

132 absence of standardization, the rehabilitation program could slightly differ in content and

133 periodization. At the time of testing the participants were allowed to return to their regular

134 sport activities (included sprinting) by the clinical staff, had recovered to their pre-injury peak

135 knee flexor torque level, and were free of any lower limb pain.

\section{2.3. $\quad \underline{\text { Protocol }}$}

138 Participants attended three sessions in a randomized order: i) a MRI session to estimate both 139 muscle volume and muscle moment arm, ii) an ultrasound session to estimate fascicle length 
and pennation angle, and iii) an experimental session during which muscle activation was

141 assessed using surface EMG. Specifically, participants sat on an isokinetic dynamometer

142 (Con-Trex, CMV AG, Dübendorf, Switzerland) with non-compliant straps placed around the

143 chest, the pelvis and the thigh. The hip and the knee were flexed at $90^{\circ}$ and $45^{\circ}$, respectively

$144\left(0^{\circ}=\right.$ neutral position for the hip and full extension for the knee). Knee angle was chosen as it

145 corresponds to the peak knee flexor torque angle, i.e., the optimal angle (23). The torque

146 signal from the isokinetic dynamometer was recorded and digitized by a USB data acquisition

147 module (DT9804; Data Translation, Marlboro, MA, USA) at $1000 \mathrm{~Hz}$. Torque was corrected

148 for gravity and low-pass filtered at $20 \mathrm{~Hz}$ using a third-order Butterworth filter. Visual

149 feedback of the exerted torque signal was displayed on a screen placed in front of the 150 participants.

151

$152 \quad$ 2.4. Estimation of muscle activation

\section{$153 \quad$ 2.4.1. Experimental tasks}

154 After a standardized warm-up (ten isometric knee flexions at $50 \%$ of peak torque and five 155 isometric knee flexions at $80 \%$ of peak torque), participants performed three MVC of the 156 knee flexors for 3 to $5 \mathrm{~s}$ with 120 -s rest in between. The maximal value obtained from a 157 moving average window of $300 \mathrm{~ms}$ was considered as the peak knee flexor torque. Then, 158 participants performed three 10 -s contractions at both $20 \%$ and $50 \%$ of MVC peak torque (30$159 \mathrm{~s}$ rest in between). This protocol was performed for each leg in a randomized order with 5 min 160 rest in between.

161

162

\subsubsection{Surface electromyography}

163 Myoelectric activity was recorded bilaterally through surface electrodes placed over the ST, $164 \mathrm{SM}$, and BF. The participants were seated on a customized piece of foam with a free space 
165 beneath each muscle to ensure that there was no contact between the electrodes and the seat.

166 We used B-mode ultrasound (v10, Aixplorer, Supersonic Imagine, Aix-en-Provence, France)

167 to determine the appropriate placement of electrodes on each muscle, longitudinally with

168 respect to the muscle fascicle's alignment and away from the borders of neighboring muscles.

169 As the superficial part of the BF short head is close to the popliteal fossa, it was not possible

170 to investigate this muscle. We therefore followed the SENIAM recommendations for

171 electrode placement on $\mathrm{BF}$ and considered the recorded myoelectrical activity originating

172 from this pair of electrodes as being representative of both the short and long head. The skin

173 was shaved and cleaned with alcohol and a pair of $\mathrm{Ag} / \mathrm{AgCl}$ electrodes (recording zone area:

$174520 \mathrm{~mm}^{2}$, Blue sensor N-00-S, Ambu, Copenhagen, Denmark) was attached to the skin with

175 an inter-electrode distance of $20 \mathrm{~mm}$ (centre-to-centre). Raw EMG signals were pre-amplified

176 (input impedance: $20 \mathrm{MM}, \mathrm{CMRR}$ : $90 \mathrm{db}$; gain: 1000), band-pass filtered (10-500 Hz, third

177 order Butterworth filter) and sampled at $2000 \mathrm{~Hz}$ (Zerowire, Aurion, Milan, Italy). EMG and

178 mechanical data were synchronized using a transistor-transistor-logic pulse recorded by a 12-

179 bit analog to digital converter (DT9804, Data Translation, Marlboro, USA).

180

181

\subsubsection{Data processing}

182 All mechanical and EMG data were analyzed using MATLAB custom-written scripts

183 (R2017a, The Mathworks, Nathick, MA, USA). The Root Mean Square (RMS) of the EMG

184 signal was calculated over a moving time window of $300 \mathrm{~ms}$ and the maximal value achieved

185 over the three trials was considered as the maximal activation level (EMG $\left.\mathrm{RMS}_{\max }\right)$. During

186 the submaximal isometric knee flexion tasks, the EMG RMS amplitude was calculated over 5

$187 \mathrm{~S}$ at the period corresponding to the lowest standard deviation of the torque signal. For each

188 trial, this value was normalized to that measured during the MVC task. The ratio of activation 
between the hamstring muscles was calculated as the normalized EMG RMS of the

190 considered muscle divided by the sum of normalized EMG RMS values of all three muscles:

$$
\text { Activation ratio (muscle) }=\frac{\% E M G R M S(\text { muscle })}{\% E M G R M S_{B F}+E M G R M S_{S M}+E M G R M S_{S T}} \times 100
$$

191

$192 \quad$ 2.5. Estimation of muscle torque-generating capacity

193 2.5.1. Magnetic resonance imaging (MRI)

194 Participants were positioned supine in the MRI scanner (MRI; 1.5 T, Intera Achieva, Philips, 195 Amsterdam, Netherlands), with their knees flexed at $45^{\circ}$. Flexible surface coils (SENSE, 196 Philips, Amsterdam, Netherlands) were strapped to the medial and lateral sides of the knee. 197 Moment arm was measured using a volumetric sequence (3D T1 fast field echo, 5.17 min, 198 FOV $250 \times 179 \mathrm{~mm}, \mathrm{TR} / \mathrm{TE}=24 / 11.5 \mathrm{~ms}$, voxel size: $1 \times 1 \times 2 \mathrm{~mm}$, flip angle: $50^{\circ}$ ) that imaged 199 the region comprised between the middle of the femur to the middle of the tibia. For each 200 muscle, the knee flexor moment arm was defined as the shortest distance between the rotation center of the knee joint and the muscle line of action using a protocol described previously (2). In short, the 3D coordinates of the lateral and medial femoral epicondyles were determined, and the center of the joint was calculated as the midpoint between these two 204 points. Then, the distal part of the hamstring muscle-tendon unit (ST, SM, BF) was outlined and the centroid of the axial slices was calculated to determine a line passing through. Then, the moment arm was considered as the shortest distance between the rotation center of the

207 joint and the musculotendon path. Note that we considered one common moment arm for both BFsh and BFlh, as their distal tendon cannot be consistently distinguished with sufficient 209 accuracy (41).

210 Muscle volume was estimated using a second MRI scan performed in a supine position, lying 211 with hips and knees fully extended. Considering that muscles are isovolumetric, joint position 212 did not affect muscle volume. A spine coil (15 elements, SENSE, Philips) was placed under 
213 the pelvis and lower limbs to perform a volumetric sequence (3D T1 turbo fast field echo,

$21413.10 \mathrm{~min}$, FOV $360 \mathrm{~mm} \times 220 \mathrm{~mm}, \mathrm{TR} / \mathrm{TE}=14 / 6.9 \mathrm{~ms}$, voxel size: $0.8 \times 0.8 \times 2 \mathrm{~mm}$, flip

215 angle: $20^{\circ}$ ). Slice thickness was $2 \mathrm{~mm}$ without an inter-slice gap. Contiguous MR images

216 were acquired from the iliac crest to half of the tibia to obtain images from the hamstring

217 heads (ST, SM, BFlh and BFsh) between their proximal and distal insertions. MR images of

218 the ST, SM, BFlh and BFsh were then segmented manually (Mimics, Materialise, Leuven,

219 Belgium; Fig. 1B) to calculate muscle volume (Fig. 1C).

\subsubsection{B-mode extended field of view ultrasound}

222 Ultrasound panoramic mode (Aixplorer V10, Supersonic Imagine) was used to estimate

223 muscle fascicle length. This technique uses an algorithm that fits a series of images, allowing

224 the entire fascicles to be scanned within one continuous scan. This approach does not require

225 extrapolating the non-visible part of the fascicle (1), resulting in a more reliable assessment of

226 muscle fascicle length compared to single B-mode images (30). Participants were lying prone

227 with the hip and the knee flexed at $90^{\circ}$ and $45^{\circ}$, respectively $\left(0^{\circ}=\right.$ neutral position for the hip

228 and full extension for the knee). An ultrasound transducer (2-10 MHz, SL10-2, Supersonic

229 Imagine, Aix-en-Provence, France) was placed over the muscle to acquire transverse images

230 along the midline to determine the musculotendon path. Then, longitudinal scans progressed

231 along this midline in the fascicle line of action at an approximate scan speed of $2 \mathrm{~cm} \cdot \mathrm{s}^{-1}$. The

232 total scan time was 10 to $15 \mathrm{~s}$, and the scan was repeated for each muscle until two images

233 with visible fascicles were obtained (Fig. 1A). A segmented line (with a spline fit) was used

234 to model the fascicle and measure its length (ImageJ v1.48, National Institutes of Health,

235 Bethesda, MD, USA). One or two fascicles were measured for the BFsh, while one fascicle

236 was measured distally, medially, and proximally for the SM and BFlh. The pennation angle 
was measured as the angle between the deep aponeurosis and the fascicle. The three values were averaged to obtain a representative value for the entire muscle.

\subsubsection{Calculation of PCSA}

The functional PCSA of each muscle was calculated as follows(32):

$$
\text { PCSA }=\frac{\text { Muscle volume }}{\text { Fascicle length }} \times \text { cosine }(\text { Pennation angle })
$$

with PCSA in $\mathrm{cm}^{2}$, muscle volume in $\mathrm{cm}^{3}$, fascicle length in $\mathrm{cm}$ and pennation angle in rad. Because ST muscle and fascicles have the same line of action (16), its PCSA was considered as the anatomical cross-sectional area measured using MRI. The ratio of PCSA was calculated as the PCSA of the considered muscle divided by the sum of the PCSA of all hamstring muscles.

\subsection{Estimation of an index of muscle torque}

We considered PCSA, EMG amplitude, and moment arm to assess the difference in torque produced by the hamstring heads. An index of muscle torque was calculated as follows:

Index of muscle torque $=P C S A \times$ moment arm $\times$ normalized $R M S E M G$ where the index of muscle torque is expressed in arbitrary units (AU), PCSA in $\mathrm{cm}^{2}$, moment arm in $\mathrm{m}$ and normalized RMS EMG in percentage of RMS $\mathrm{EMG}_{\max }$. The torque ratio was calculated as the index of torque of the considered muscle divided by the sum of the index of torque of all three muscles.

\section{7. $\quad$ Statistics}

Statistical analyses were performed using Statistica (v8, Statsoft, Tulsa, OK, USA). Distributions consistently passed the Kolmogorov-Smirnov normality test, and all data are reported as mean $\pm \mathrm{SD}$. MVC peak torque was compared between the uninjured and injured 
260 limb using a Student paired t-test. The effect of previous injury on RMS EMG values was

261 tested using a repeated-measures three-way ANOVA (within-subject factors: intensity [20\%

262 and 50\% MVC], limb [uninjured, injured] and muscle [ST, SM, BF]). The effect of a previous

263 injury on muscle volume and PCSA was assessed using repeated-measures two-way

264 ANOVAs (within-subject factors: limb [uninjured, injured] and muscle [ST, SM, BF]). When

265 the sphericity assumption in repeated measures ANOVAs was violated (Mauchly's test), a

266 Geisser-Greenhouse correction was used. When appropriate, post-hoc analyses were

267 performed using the Bonferroni test. To address the main aim of the study, we compared 268 muscle activation, PCSA and torque ratios (BF/Hams, SM/Hams, ST/Hams) between limbs 269 using separated Student paired t-tests as the independence principle of the ANOVA was not 270 respected. The level of significance was set at $P<0.05$.

271

272 3. RESULTS

273 Torque data

274 Peak MVC torque did not significantly differ between limbs $(164.3 \pm 37.8 \mathrm{Nm}$ and $171.3 \pm 28.5$

$275 \mathrm{Nm}$ for the injured and uninjured limb, respectively; $P=0.20$ ). In turn, submaximal torque 276 targets were similar between limbs at both $20 \%$ of MVC $(32.9 \pm 7.6 \mathrm{Nm}$ and $34.3 \pm 5.7 \mathrm{Nm}$ for 277 the injured and uninjured limb, respectively) and 50\% of MVC $(82.1 \pm 18.9 \mathrm{Nm}$ and $85.7 \pm 14.2$ $278 \mathrm{Nm}$ for the injured and uninjured limb, respectively).

\section{$\underline{\text { Muscle activation }}$}

281 A main effect of intensity $(P<0.001)$ was observed on muscle activation, with a mean 282 hamstring activation of $14.8 \pm 7.0 \%$ at $20 \% \mathrm{MVC}$ and $38.3 \pm 13.3 \%$ at $50 \% \mathrm{MVC}$ (data for 283 each individual muscle are detailed in Table 2). There was neither a main effect of limb $284(P=0.85)$ nor a main effect of muscle $(P=0.48)$ on muscle activation. In addition, there was 
no significant interactions between intensity and limb $(P=0.39)$, intensity and muscle

$286(P=0.41)$, limb and muscle $(P=0.14)$ and intensity, limb and muscle $(P=0.95)$.

287 The activation ratios measured during the isometric contraction performed at $20 \% \mathrm{MVC}$ are

288

289

290

291

292

293

294

295

296

297

298

299

300

301

302

depicted in Fig. 2A. We observed a higher SM/Hams ratio for the injured limb $(38.2 \pm 11.0 \%)$ compared to the uninjured limb $(34.3 \pm 10.8 \%, P=0.018)$. No between-limb differences were observed for $\mathrm{BF} / \mathrm{Hams}(P=0.10)$ and $\mathrm{ST} / \mathrm{Hams}(P=0.91)$. At $50 \% \mathrm{MVC}$, all ratios were comprised between $30.3 \pm 7.6 \%$ (BF/Hams of the injured limb) and $35.4 \pm 6.9 \%$ (ST/Hams of the injured limb; Fig. 2B), with no significant between-limb differences [BF/Hams $(P=0.27)$, $\mathrm{SM} / \operatorname{Hams}(P=0.12)$, and ST/Hams $(P=0.90)]$.

\section{Force-generating capacity}

Although we observed a significant main effect of muscle $(P<0.001)$ on volume, there was neither a main effect of limb $(P=0.20)$ nor an interaction between limb and muscle $(P=0.08)$. BF volume was significantly larger than SM $(P<0.001)$ and ST $(P<0.001)$, with no differences between SM and ST $(P=0.34)$.

Regarding PCSA, we found a significant main effect of muscle $(P<0.001)$, with no effect of limb $(P=0.38)$. There was a significant interaction between limb and muscle $(P=0.032)$.

Regardless of the limb, PCSA was smaller for ST compared to both BF $(P<0.001$ and $P<0.001$ on injured and uninjured limbs, repsectively) and SM $(P<0.001$ and $P<0.001$ on injured and uninjured limbs, repsectively). In addition, BF exhibited larger PCSA than SM ( $P=0.031$ and $P<0.001$ on injured and uninjured limb, respectively). Note that we ran the same analysis including BFlh and BFsh heads, and we did not observe a significant interaction between limb and muscle $(P=0.063)$.

The BF/Hams ratio for PCSA was $-3.0 \pm 6.2 \%$ lower in the injured limb than in the uninjured limb $(P=0.045)$. This difference was observed in 12 out of $17(71 \%)$ of the participants as 
reflected by the individual data (Fig. 3). Inversely, there was a trend, albeit non significant,

311 for the SM/Hams ratio to be higher in the injured side compared to the uninjured side

$312(P=0.083)$. No between-limb differences were observed for ST/Hams $(P=0.661)$.

313

\section{Bilateral differences in muscle coordination}

315 When ANOVA was applied on the index of muscle torque, we observed a significant main

316 effect of intensity $(P<0.001)$ and muscle $(P=0.005)$, a significant interaction between

317 intensity and muscle $(P=0.016)$, and a significant interaction between limb and muscle

$318(P=0.022)$. There was neither a main effect of limb $(P=0.88)$ nor an interaction between

319 intensity and limb $(P=0.57)$. For the sake of clarity, we report only the statistics associated

320 with the interaction between muscle and limb, which relates to the main aim of this study.

321 Regardless of the limb, ST produced a lower torque than both SM $(P=0.006$ for both limbs $)$

322 and $\mathrm{BF}(P<0.001$ for both limbs $)$. The torque produced by $\mathrm{BF}$ was higher than that produced

323 by $\mathrm{SM}$ in the uninjured limb $(P=0.038)$, while no difference was observed between these two

324 muscles in the injured limb $(P=1.00)$.

325 We considered muscle coordination as the distribution of torque among the three heads of the

326 hamstring muscles. The contribution of $\mathrm{BF}$ torque over the total hamstring torque (BF/Hams)

327 was lower in the injured than in the uninjured limb at $20 \% \mathrm{MVC}(-10.8 \pm 27.5 \% ; P=0.038$;

328 Fig. 4A). Inversely, the contribution of SM (SM/Hams) was higher in the injured than in the

329 uninjured limb $(+17.2 \pm 27.4 \% ; P=0.007$; Fig. 4A). No between-limb differences were

330 observed for ST/Hams. Notably, 13 participants $(76 \%)$ presented a lower $\mathrm{BF} / \mathrm{Hams}$ ratio

331 associated with a higher $\mathrm{SM} / \mathrm{Hams}$ ratio in the injured than in the uninjured $\operatorname{limb}$. At $50 \%$ of

332 MVC, only SM/Hams was higher in the injured compared to injured limb $(+12.5 \pm 21.3 \%$;

$333 P=0.035)$. No significative differences were observed for $\mathrm{BF} / \mathrm{Hams}(P=0.13)$ and $\mathrm{ST} / \mathrm{Hams}$

$334 \quad(P=0.92$; Fig. 4B). 


\section{DISCUSSION}

337 This study aimed to determine whether coordination between hamstring muscles differs

338 between an injured and uninjured limb in elite athletes with a history of unilateral hamstring 339 strain injury. Our experimental approach considered muscle activation measured during an 340 isometric task, muscle PCSA, and muscle moment arm to estimate an index of torque for each 341 muscle. Our results highlight different coordination strategies between limbs, with a lower

342 contribution of the injured muscle $(\mathrm{BF})$ to total knee flexion torque compared to the uninjured 343 limb. This reduced contribution of BF was compensated by a higher contribution of the SM 344 muscle in the injured limb. These changes observed in the injured limb resulted from changes 345 in activation of SM and/or the muscle force-generating capacity of BF muscle. These specific 346 adaptations were observed after the completion of rehabilitation and when the participants no 347 longer reported pain and were able to sprint. These results have clinical relevance as they 348 provide evidence that substantial bilateral differences in hamstring coordination persist at the 349 return to regular training. According to pain and injury adaptation theories, these changes may 350 have long-term negative consequences.

351

\section{$352 \quad$ Methodological considerations}

353 Some methodological considerations should be kept in mind when interpreting the present 354 data. First, muscle activation was assessed using surface EMG in a bipolar configuration. In 355 order to minimize crosstalk, we used B-mode ultrasound to ensure similar electrode locations 356 between participants, away from the border of neighboring muscles and aligned with the 357 fascicle line of action. In a recent study, we showed that this procedure provides reliable 358 measurements of activation between days (2). The normalization procedure is also crucial to 359 accurately compare activation level between muscles and participants. Using the twitch 
interpolation method, previous studies have reported that young healthy participants are able

361 to achieve near-complete activation of their hamstrings (e.g., $98.4 \pm 0.9 \%$ in Kirk et al. (24)).

362 Also, we found similar MVC torque values between legs in this study. We can therefore

363 reasonably assume that the hamstring muscles of both legs were fully activated during the

364 maximal isometric contractions.

365 Second, although we considered two important mechanical factors (i.e., PCSA and moment

366 arm), which influence torque-generating capacity during submaximal isometric contractions,

367 we did not consider specific tension or the individual muscle force-length relationship.

368 However, to date, there is no experimental technique available to accurately measure these

369 mechanical factors for the hamstrings. In addition, specific tension varies only marginally

370 between muscles with similar fiber type composition (14), especially at low contraction

371 intensity during which type I fibers are preferentially recruited. Given that hamstring muscles

372 share a similar function (39) and that the force-length properties of human skeletal muscles

373 may reflect the requirements imposed by daily activities (19), we considered each muscle as

374 acting at a comparable length relative to their optimal length.

375 Third, as our experimental tasks involved isometric contractions, our results cannot be

376 extrapolated to dynamic tasks. Of note, accurate estimation of force during dynamic tasks

377 remains challenging, if not impossible. Although musculoskeletal modeling may provide an

378 estimation of individual muscle forces during dynamic tasks, use of such modeling is limited

379 within the context of muscle injury. This is because most of the models make an a priori

380 assumption that muscles forces are optimally redistributed after injury (34), which is not

381 necessarily true. Recent evidence demonstrates that adaptations in muscle coordination are

382 not predictable as they do not follow any optimization rules or a stereotypical response (10,

383 20, 31). Changes in muscle coordination can occur on the uninjured limb even after a

384 unilateral alteration of force-generating capacity, mostly because of changes in motor control 
385 in both limbs $(5,6)$. Such a cross-sectional design therefore precludes the possibility of

386 considering coordination of the contralateral limb as a 'pre-injury' status and in turn prevents

387 us from making any conclusions regarding a causal association between injury and muscle

388 coordination observed in the injured limb. With these considerations in mind, we interpreted

389 the differences in the hamstring coordination as between-limb differences rather than post-

390 injury adaptations.

391

392 Bilateral differences in muscle activation

393 Theories about motor adaptation to pain and injury have proposed that movement is altered in 394 order to decrease the threat of further pain or reinjury (20). The only way for the central 395 nervous system to adapt movement is to alter muscle activation. Our results did not show 396 significant differences in the activation of the injured (BF) muscle compared to the uninjured 397 limb (Table 2). Previous research has also suggested that BF activation is reduced or 398 unchanged following injury $(28,38)$. Such results were obtained during eccentric maximal 399 contractions that involve a specific neural control more prone to elicit neuromuscular 400 inhibition at both the supraspinal and spinal levels compared to concentric or isometric tasks 401 (12). Alternatively, these discrepancies may reflect that injury may not only alter the 402 activation of the injured muscle but also the relative contribution of other muscle synergists, 403 as a compensatory mechanism against neuromuscular inhibition (9). Here, we focused on the 404 muscle activation ratio to estimate the contribution of each muscle head to total hamstring 405 activation. Given that hamstring muscles have redundant contributions to knee flexor torque, 406 submaximal isometric contractions could be achieved using multiple combinations of 407 muscles. We observed an increased contribution of the activation of an uninjured synergist 408 muscle (SM), which is likely compensating for a decreased contribution in activation from the 409 injured muscle $(\mathrm{BF})$, albeit non-significant (bilateral difference in $\mathrm{BF} / \mathrm{Hams}: P=0.10$ ). Of 
note, a lower BF/Hams ratio was observed in the injured limb in 11 out of 17 participants.

411 Changes in the ratios of muscle activation have also been observed during a Nordic hamstring

412

413

414

415

416

417 exercise performed by previously injured athletes(4). Specifically, they found a greater contribution of the $\mathrm{BF}$ in total hamstring activation during the late phase of the Nordic hamstring, which is not consistent with our results. However, it is difficult to interpret these changes regarding the injury because the injured muscle was not specified. The Nordic hamstring is an eccentric-biased (i.e., with a specific neural control) bilateral near-maximal task, which offers less degree of freedom to change muscle activation.

The differences in activation ratios among hamstring muscles can be discussed within the context of current motor control theories. The optimal feedback control theory suggests that the activation strategies adopted by the central nervous system aim to minimize a cost and/or maximize a benefit (40). In the context of pain and injury, unloading the injured muscle, as suggested by previous studies $(28,38)$, can be considered as a benefit. This unloading was compensated with an increased SM activation, which seems to be an efficient strategy. Indeed, the metabolic cost associated with force generation is related to the activated volume of muscle to generate a given force. Given that muscle force is generally proportional to the cross-sectional area of activated fibers, longer-fibered muscles require a larger activated volume to generate a given force (3). This means that the SM may have a lower ATP consumption per unit of force generated compared to the ST. Therefore, differences in $\mathrm{SM} /$ Hams activation ratios may result from an optimization process initiated by the central nervous system at the time of injury $(10,20)$. Alternatively, each individual might use 'motor habits', i.e., a set of valid distributions of activations to perform the task without necessarily minimizing cost $(25,31)$. In the context of muscle injury, the distribution of activations might result from a rescaling of the original muscle activity, which is not reoptimized despite the deficit in force-generating capacity observed in the injured muscle (31). This could explain 
why some participants (6 out of 17) did not exhibit any change in BF/Hams activation ratios. At $50 \%$ of MVC, activation ratios were not different between legs, likely because a higher activation of the hamstring muscles is required to perform the task $(8,21)$. During such tasks, fewer degrees of freedom are available to modify the activation distribution while maintaining the goal of the task.

\section{Coupling between muscle activation and PCSA differences}

Despite a similar PCSA for the whole hamstring group between limbs, we found that the $\mathrm{BF} /$ Hams ratio of PCSA was lower in the injured limb than in the uninjured limb $(P=0.045)$. In other words, the previously injured muscle accounted for a lower proportion of the total hamstring PCSA. An opposite trend (albeit non-significant) was observed for SM $(P=0.08)$. The observed reduction in PCSA seems more likely attributable to a reduction in the volume of $\mathrm{BF}$ as reflected by the similar relative differences in both parameters and the lack of changes in pennation angle (Table 3). This is constant with previous findings of selective atrophy of the BFlh up to 23 months after injury (37) or at 6 months after the return to play (33). Note that the BF was the injured muscle in most of the participants ( 72 to $85 \%$ ) in the later studies $(33,37)$.

Due to its cross-sectional design, the present study cannot determine whether the observed bilateral differences in both muscle activation and PCSA distribution is a contributing factor or result from injury. For example, it is possible that a prolonged reduction in activation might result in the atrophy of the injured muscle, even after a rehabilitation program. Subsequently, the volume of the non-activated part of the muscle might decrease due to insufficient mechanical stimuli, resulting in atrophy of the whole muscle. Alternatively, these differences in activation and PCSA between the legs could have been present before the injury. However, asymmetry in hamstring volume has not been reported for active people (2) or sprinters with 
no previous injury (17). Moreover, we observed similar hamstring activation ratios across

461 legs during submaximal isometric knee flexion in healthy controls (2). Thus, between-limb 462 differences in activation and PCSA have only been reported in previously-injured athletes,

463 which suggest that the injury might be the cause of such alterations $(33,37)$. Further 464 prospective investigations are needed to test this assumption.

466 Individual hamstring coordination and their functional consequences

467 Our results provide strong evidence of different force-sharing strategies in an injured versus 468 an uninjured limb. Adaptations in muscle coordination after a hamstring injury have been 469 suggested, using indirect measures such as functional MRI $(35,36)$ and surface EMG $(4,9)$. 470 Here the index of muscle torque provided a more direct assessment of muscle coordination 471 than activation alone (22). At $20 \%$ of $\mathrm{MVC}$, we found the $\mathrm{BF} / \mathrm{Hams}$ torque ratio to be lower $472(-10.8 \pm 20.7 \%)$ and the SM/Hams torque ratio higher $(+17.2 \pm 27.4 \%)$ in the injured than in the 473 non-injured limb. A large majority of participants adopted this strategy (13 out 17 474 participants). Although the origin of such differences remains unknown, it might have 475 functional consequences. A force deficit in the injured muscle could decrease its capacity to 476 sustain high mechanical loading, and in turn increase its susceptibility to damage (27). Data 477 from animal models have also shown that the greater the force produced by a muscle, the 478 higher its energy absorption before failure and injury (15).

479 Therefore, strengthening the injured muscle could be a primary target of rehabilitation 480 programs to adjust toward a balanced contribution of hamstring heads to total torque in order 481 to reduce the risk of reinjury. Crossley et al. (7) have shown that muscle coordination could 482 be durably changed in patients suffering from patellofemoral pain using an appropriate 483 rehabilitation program. In addition, recent studies demonstrated muscle- and regional-specific 484 activations within hamstring in response to various strengthening exercises (18). For instance, 
485 hip extension or flywheel curl exercises could be proposed to selectively activate and 486 strengthen BF muscle. Whether the chronic effects elicited by such individualized training 487 could participate to level the contribution of the pre-injured muscle to total hamstring torque 488 remains to be investigated. These research questions open promising perspectives for well489 trained athletes (as those included in the present study) particularly exposed to the detrimental 490 effects of hamstring strain injuries.

491

\section{5. CONCLUSION}

493 Previously injured athletes have bilateral differences in hamstring coordination. During 494 submaximal knee flexions performed at $20 \%$ of MVC, the injured BF muscle contributed less 495 to the total knee flexor torque than the same muscle in the uninjured limb; and this was compensated by a larger contribution of the SM muscle, also observed at $50 \%$ of MVC. These

497 changes in muscle coordination were attributed to changes in muscle force-generating 498 capacity and/or activation. These bilateral differences in hamstring coordination raises the question of its long-term impact on hamstring morphology and mechanics. Further studies are required to determine whether these adaptations to initial injury could constitute an intrinsic risk factor that contributes to the high rate of hamstring injury recurrence.

502

\section{ACKNOWLEDGMENTS:}

504 The authors thank J. Renoux and M. Crema for assistance with MRI data collection. 505 506

\section{GRANTS:}

507 S. Avrillon was supported by a scholarship funded by the French Ministry of Research. F. 508 Hug was supported by a fellowship from the Institut Universitaire de France (IUF). 


\section{DISCLOSURES:}

511 No conflicts of interest, financial or otherwise, are declared by the authors. Authors declare

512 that they have no conflicts of interest relevant to the content of this original research article.

513

514 


\section{TABLES:}

516 Table 1- Demographics and injury characteristics of study participants. The grade refers to 517 the classification of the Munich consensus statement. Injury-to-test time represents the 518 number of days between the injury occurrence and the experiment. BFlh: Biceps femoris long 519 head. ST: Semitendinosus

\begin{tabular}{|c|c|c|c|c|c|c|c|}
\hline $\begin{array}{l}\text { Partici } \\
\text { pant }\end{array}$ & $\begin{array}{l}\text { Age } \\
\text { (yr) }\end{array}$ & $\begin{array}{l}\text { Height } \\
\text { (m) }\end{array}$ & $\begin{array}{c}\text { Body } \\
\text { mass (kg) }\end{array}$ & $\begin{array}{l}\text { Injury site } \\
\text { (side) }\end{array}$ & Grade & $\begin{array}{c}\text { Rehabilitation } \\
\text { duration (days) }\end{array}$ & $\begin{array}{c}\text { Injury-to-test } \\
\text { time (days) }\end{array}$ \\
\hline 1 & 25 & 1.78 & 65 & $\begin{array}{l}\text { BFlh } \\
\text { (Right) }\end{array}$ & 2 & 21 & 41 \\
\hline 2 & 25 & 1.78 & 74 & $\begin{array}{l}\text { BFlh } \\
\text { (Left) }\end{array}$ & 3 & 42 & 141 \\
\hline 3 & 24 & 1.78 & 68 & $\begin{array}{l}\text { BFlh } \\
\text { (Right) }\end{array}$ & 2 & 21 & 40 \\
\hline 4 & 26 & 1.85 & 89 & $\begin{array}{l}\text { BFlh } \\
\text { (Right) }\end{array}$ & 2 & 14 & 62 \\
\hline 5 & 38 & 1.84 & 70 & $\begin{array}{l}\text { BFlh/ST } \\
\text { (Left) }\end{array}$ & 2 & 21 & 69 \\
\hline 6 & 33 & 1.84 & 77 & $\begin{array}{l}\text { BFlh } \\
\text { (Right) }\end{array}$ & 2 & 28 & 57 \\
\hline 7 & 33 & 1.89 & 90 & $\begin{array}{l}\text { BFlh } \\
\text { (Right) }\end{array}$ & 2 & 21 & 113 \\
\hline 8 & 24 & 1.84 & 86 & $\begin{array}{l}\text { BFlh } \\
\text { (Left) }\end{array}$ & 2 & 14 & 82 \\
\hline 9 & 33 & 1.72 & 70 & $\begin{array}{l}\text { BFlh } \\
\text { (Right) }\end{array}$ & 2 & 56 & 183 \\
\hline 10 & 27 & 1.79 & 73 & $\begin{array}{l}\text { BFlh } \\
\text { (Right) }\end{array}$ & 2 & 35 & 102 \\
\hline 11 & 20 & 1.78 & 72 & $\begin{array}{l}\text { BFlh } \\
\text { (Right) }\end{array}$ & 2 & 14 & 94 \\
\hline 12 & 31 & 1.70 & 69 & $\begin{array}{l}\text { BFlh/ST } \\
\text { (Right) }\end{array}$ & 3 & 42 & 78 \\
\hline 13 & 23 & 1,80 & 69 & $\begin{array}{l}\text { BFlh } \\
\text { (Left) }\end{array}$ & 2 & 42 & 113 \\
\hline 14 & 23 & 1,84 & 83 & $\begin{array}{l}\text { BFlh } \\
\text { (Left) }\end{array}$ & 2 & 28 & 83 \\
\hline 15 & 18 & 1,73 & 65 & $\begin{array}{l}\text { BFlh } \\
\text { (Right) }\end{array}$ & 2 & 63 & 198 \\
\hline 16 & 21 & 1,75 & 68 & $\begin{array}{l}\text { BFlh } \\
\text { (Right) }\end{array}$ & 3 & 70 & 192 \\
\hline 17 & 23 & 1,78 & 78 & $\begin{array}{l}\text { BFlh/ST } \\
\text { (Right) }\end{array}$ & 2 & 21 & 22 \\
\hline
\end{tabular}


522 Table 2. Normalized EMG RMS measured in injured and uninjured limb during 523 submaximal isometric knee flexions performed at $20 \%$ and $50 \%$ of the peak torque 524 produced during maximal voluntary contraction (MVC). BF, biceps femoris; SM, 525 semimembranosus; ST, semitendinosus.

\section{INJURED LIMB $\quad$ UNINJURED LIMB}

\begin{tabular}{l|cccccc}
\hline & BF & SM & ST & BF & SM & ST \\
& $(\% \max )$ & $(\% \max )$ & $(\% \max )$ & $(\% \max )$ & $(\% \max )$ & $(\% \max )$ \\
$\mathbf{2 0 \%}$ MVC & $13.0 \pm 6.0$ & $18.0 \pm 8.2$ & $14.8 \pm 7.2$ & $14.1 \pm 7.6$ & $15.4 \pm 8.0$ & $13.5 \pm 4.4$ \\
$\mathbf{5 0 \%}$ MVC & $34.7 \pm 12.8$ & $39.9 \pm 14.5$ & $39.6 \pm 9.5$ & $37.7 \pm 15.6$ & $38.5 \pm 17.7$ & $39.5 \pm 9.1$
\end{tabular}

526

527

528 
529 Table 3 - Muscle architecture. Fascicle length, pennation angle, muscle volume, 530 physiological cross-sectional area (PCSA) and moment arm for injured and uninjured limb. 531 Statistics are only reported for muscle $\times$ limb interaction.

$532{ }^{a}$ Indicates a significant difference with $B F,{ }^{b}$ indicates a significant difference with $S M$ and ${ }^{c}$ 533 indicates a significant difference with ST.

INJURED LIMB $\quad$ UNINJURED LIMB

\begin{tabular}{|c|c|c|c|c|c|c|c|c|}
\hline & BFsh & BFIh & SM & ST & BFsh & BFlh & SM & ST \\
\hline FL (CM) & $11.9 \pm 1.7$ & $11.9 \pm 1.0$ & $9.8 \pm 1.1$ & & $11.9 \pm 1.1$ & $11.8 \pm 1.7$ & $9.9 \pm 1.3$ & \\
\hline \multirow[t]{2}{*}{ PA $\left({ }^{\circ}\right)$} & $14.1 \pm 3.0$ & $9.4 \pm 1.0$ & $11.6 \pm 2.1$ & & $13.3 \pm 2.5$ & $10.1 \pm 1.6$ & $11.4 \pm 2.0$ & \\
\hline & \multicolumn{2}{|c|}{$\mathbf{B F}$} & SM & ST & \multicolumn{2}{|c|}{ BF } & SM & ST \\
\hline $\begin{array}{l}\text { VOLUME } \\
\qquad\left(\mathbf{C M}^{3}\right)\end{array}$ & \multicolumn{2}{|c|}{$433.3 \pm 75.0$} & $333.2 \pm 78.8$ & $355.8 \pm 89.9$ & \multicolumn{2}{|c|}{$439.8 \pm 73.2$} & $320.8 \pm 68.1$ & $346.3 \pm 83.1$ \\
\hline $\begin{array}{l}\text { PCSA } \\
\left(\mathrm{CM}^{2}\right)\end{array}$ & \multicolumn{2}{|c|}{$35.9 \pm 7.4^{b c}$} & $33.7 \pm 8.4^{\mathrm{ac}}$ & $19.2 \pm 4.8^{\mathrm{ab}}$ & \multicolumn{2}{|c|}{$36.9 \pm 7.7^{b c}$} & $32.2 \pm 7.3^{\mathrm{ac}}$ & $18.9 \pm 5.2^{b c}$ \\
\hline MOMENT & \multirow{2}{*}{\multicolumn{2}{|c|}{$5.0 \pm 0.3$}} & & & \multirow{2}{*}{\multicolumn{2}{|c|}{$4.9 \pm 0.4$}} & & \\
\hline $\begin{array}{l}\text { ARM } \\
(\mathrm{CM})\end{array}$ & & & $4.9 \pm 0.5$ & $5.8 \pm 0.6$ & & & $4.8 \pm 0.5$ & $5.8 \pm 0.7$ \\
\hline
\end{tabular}

534

535 


\section{FIGURES}

537 Figure 1 - Individual example of muscle architecture measurements. A. Panoramic 538 ultrasound image of the biceps femoris long head (BFlh) muscle. This image was used to 539 calculate BFlh fascicle length. The yellow arrows indicate a fascicle. B. Individual example

540 of MRI slice where each muscle was segmented. BFsh, biceps femoris short head; BFlh, 541 biceps femoris long head; SM, semimembranosus; ST, semitendinosus. The volumes of all 542 slices were then summed to obtain muscle volume (SM on panel C.)

543 Figure 2 - Ratios of activation for hamstring muscles for the uninjured (black scatters) and 544 injured (white scatters) limb. The ratios of EMG RMS were estimated during submaximal 545 isometric knee flexions performed at $20 \%$ and $50 \%$ of the peak torque produced during 546 maximal voluntary contraction (MVC). * Indicates a significant difference between limb $547(P<0.05)$.

548 Figure 3 - Ratios of physiological cross-sectional area (PCSA) for the uninjured (black 549 scatters) and injured (white scatters) limb. * Indicates a significant difference between limbs $550 \quad(P>0.05)$.

551 Figure 4 - Torque ratios for the uninjured (black scatters) and injured (white scatters)

552 limb. The ratios of torque were estimated during submaximal isometric knee flexions 553 performed at $20 \%$ and $50 \%$ of the peak torque produced during maximal voluntary 554 contraction (MVC).

$555 *$ Indicates a significant difference between limbs $(P<0.05)$. 
558 1. Adkins AN, Franks PW, Murray WM. Demonstration of extended field-of-view

559

560

561

562

563

564

565

566

567

568

569

570

571

572

573

574

575

576

577

578

579

580

581

582

583

584

585

586

587

588

589

590

591

592

593

594

ultrasound's potential to increase the pool of muscles for which in vivo fascicle length is measurable. J Biomech 63: 179-185, 2017.

2. Avrillon S, Guilhem G, Barthelemy A, Hug F. Coordination of hamstrings is individual specific and is related to motor performance. J Appl Physiol (1985) 125: 1069-1079, 2018.

3. Biewener AA. Locomotion as an emergent property of muscle contractile dynamics. $J$ Exp Biol 219: 285-94, 2016.

4. Blandford L, Theis $\mathbf{N}$, Charvet I, Mahaffey $\mathbf{R}$. Is neuromuscular inhibition detectable in elite footballers during the Nordic hamstring exercise? Clin Biomech (Bristol, Avon) 58: 39-43, 2018.

5. Brochner Nielsen NP, Hug F, Guevel A, Fohanno V, Lardy J, Dorel S. Motor adaptations to unilateral quadriceps fatigue during a bilateral pedaling task. Scan J Med Sci Sports 27: 1724-1738, 2017.

6. Carroll TJ, Herbert RD, Munn J, Lee M, Gandevia SC. Contralateral effects of unilateral strength training: evidence and possible mechanisms. J Appl Physiol (1985) 101: 1514-22, 2006.

7. Crossley K, Bennell K, Green S, Cowan S, McConnell J. Physical therapy for patellofemoral pain: a randomized, double-blinded, placebo-controlled trial. Am J Sports Med 30: 857-65, 2002.

8. Crouzier M, Lacourpaille L, Nordez A, Tucker K, Hug F. Neuromechanical coupling within the human triceps surae and its consequence on individual force-sharing strategies. J Exp Biol 221, 2018.

9. Daly C, McCarthy Persson U, Twycross-Lewis R, Woledge RC, Morrissey D. The biomechanics of running in athletes with previous hamstring injury: A case-control study. Scan J Med Sci Sports 26: 413-20, 2016.

10. van Dieen JH, Flor H, Hodges PW. Low-Back Pain Patients Learn to Adapt Motor Behavior With Adverse Secondary Consequences. Exerc Sport Sci Rev 45: 223-229, 2017.

11. Dimmick S, Linklater JM. Imaging of Acute Hamstring Muscle Strain Injuries. Semin Musculoskelet Radiol 21: 415-432, 2017.

12. Duchateau J, Enoka RM. Neural control of lengthening contractions. J Exp Biol 219: 197-204, 2016.

13. Fyfe JJ, Opar DA, Williams MD, Shield AJ. The role of neuromuscular inhibition in hamstring strain injury recurrence. J Electromyogr Kinesiol 23: 523-30, 2013.

14. Garrett WE, Califf JC, Bassett FH. Histochemical correlates of hamstring injuries. Am J Sports Med 12: 98-103, 1984. 
15. Garrett WE, Safran MR, Seaber AV, Glisson RR, Ribbeck BM. Biomechanical comparison of stimulated and nonstimulated skeletal muscle pulled to failure. Am J Sports Med 15: 448-54, 1987.

16. Haberfehlner H, Maas H, Harlaar J, Becher JG, Buizer AI, Jaspers RT. Freehand three-dimensional ultrasound to assess semitendinosus muscle morphology. J Anat 229: 591-9, 2016.

17. Handsfield GG, Knaus KR, Fiorentino NM, Meyer CH, Hart JM, Blemker SS. Adding muscle where you need it: non-uniform hypertrophy patterns in elite sprinters. Scan J Med Sci Sports 27: 1050-1060, 2017.

18. Hegyi A, Csala D, Peter A, Finni T, Cronin NJ. High-density electromyography activity in various hamstring exercises. Scan J Med Sci Sports 29: 34-43, 2019.

19. Herzog W, Guimaraes AC, Anton MG, Carter-Erdman KA. Moment-length relations of rectus femoris muscles of speed skaters/cyclists and runners. Med Sci Sports Exerc 23: 1289-96, 1991.

20. Hodges PW, Tucker K. Moving differently in pain: a new theory to explain the adaptation to pain. Pain 152: S90-8, 2011.

21. Hug F, Goupille C, Baum D, Raiteri BJ, Hodges PW, Tucker K. Nature of the coupling between neural drive and force-generating capacity in the human quadriceps muscle. Proc Biol Sci 282, 2015.

22. Hug F, Tucker K. Muscle Coordination and the Development of Musculoskeletal Disorders. Exerc Sport Sci Rev 45: 201-208, 2017.

23. Kilgallon M, Donnelly AE, Shafat A. Progressive resistance training temporarily alters hamstring torque-angle relationship. Scan J Med Sci Sports 17: 18-24, 2007.

24. Kirk EA, Gilmore KJ, Rice CL. Neuromuscular changes of the aged human hamstrings. J Neurophysiol 120: 480-488, 2018.

25. Loeb GE. Optimal isn’t good enough. Biol Cybern 106: 757-65, 2012.

26. Lund JP, Donga R, Widmer CG, Stohler CS. The pain-adaptation model: a discussion of the relationship between chronic musculoskeletal pain and motor activity. Can $J$ Physiol Pharmacol 69: 683-94, 1991.

27. Opar DA, Williams MD, Shield AJ. Hamstring strain injuries: factors that lead to injury and re-injury. Sports Med 42: 209-26, 2012.

28. Opar DA, Williams MD, Timmins RG, Dear NM, Shield AJ. Knee flexor strength and bicep femoris electromyographical activity is lower in previously strained hamstrings. J Electromyogr Kinesiol 23: 696-703, 2013.

29. Orchard JW, Seward H, Orchard JJ. Results of 2 decades of injury surveillance and public release of data in the Australian Football League. Am J Sports Med 41: 734-41, 2013. 
651

652

653

654

655

656

657

658

659

660

661

662

663

664
30. Pimenta R, Blazevich AJ, Freitas SR. Biceps Femoris Long-Head Architecture Assessed Using Different Sonographic Techniques. Med Sci Sports Exerc 50: 2584 2594, 2018.

31. de Rugy A, Loeb GE, Carroll TJ. Muscle coordination is habitual rather than optimal. J Neurosci 32: 7384-91, 2012.

32. Sacks RD, Roy RR. Architecture of the hind limb muscles of cats: functional significance. J Morphol 173: 185-95, 1982.

33. Sanfilippo JL, Silder A, Sherry MA, Tuite MJ, Heiderscheit BC. Hamstring strength and morphology progression after return to sport from injury. Med Sci Sports Exerc 45: 448-54, 2013.

34. Schache AG, Kim HJ, Morgan DL, Pandy MG. Hamstring muscle forces prior to and immediately following an acute sprinting-related muscle strain injury. Gait \& Posture 32: 136-40, 2010.

35. Schuermans J, Van Tiggelen D, Danneels L, Witvrouw E. Biceps femoris and semitendinosus--teammates or competitors? New insights into hamstring injury mechanisms in male football players: a muscle functional MRI study. Br J Sports Med 48: 1599-606, 2014.

36. Schuermans J, Van Tiggelen D, Danneels L, Witvrouw E. Susceptibility to Hamstring Injuries in Soccer: A Prospective Study Using Muscle Functional Magnetic Resonance Imaging. Am J Sports Med 44: 1276-85, 2016.

37. Silder A, Heiderscheit BC, Thelen DG, Enright T, Tuite MJ. MR observations of long-term musculotendon remodeling following a hamstring strain injury. Skeletal Radiol 37: 1101-9, 2008.

38. Sole G, Milosavljevic S, Nicholson HD, Sullivan SJ. Selective strength loss and decreased muscle activity in hamstring injury. J Orthop Sports Phys Ther 41: 354-63, 2011.

39. Stepien K, Smigielski R, Mouton C, Ciszek B, Engelhardt M, Seil R. Anatomy of proximal attachment, course, and innervation of hamstring muscles: a pictorial essay. Knee Surg Sports Traumatol Arthrosc 27: 673-684, 2019.

40. Todorov E, Jordan MI. Optimal feedback control as a theory of motor coordination. Nature Neurosci 5: 1226-35, 2002.

41. Woodley SJ, Mercer SR. Hamstring muscles: architecture and innervation. Cells, Tissues, Organs 179: 125-41, 2005. 


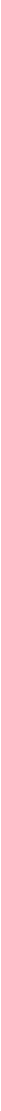



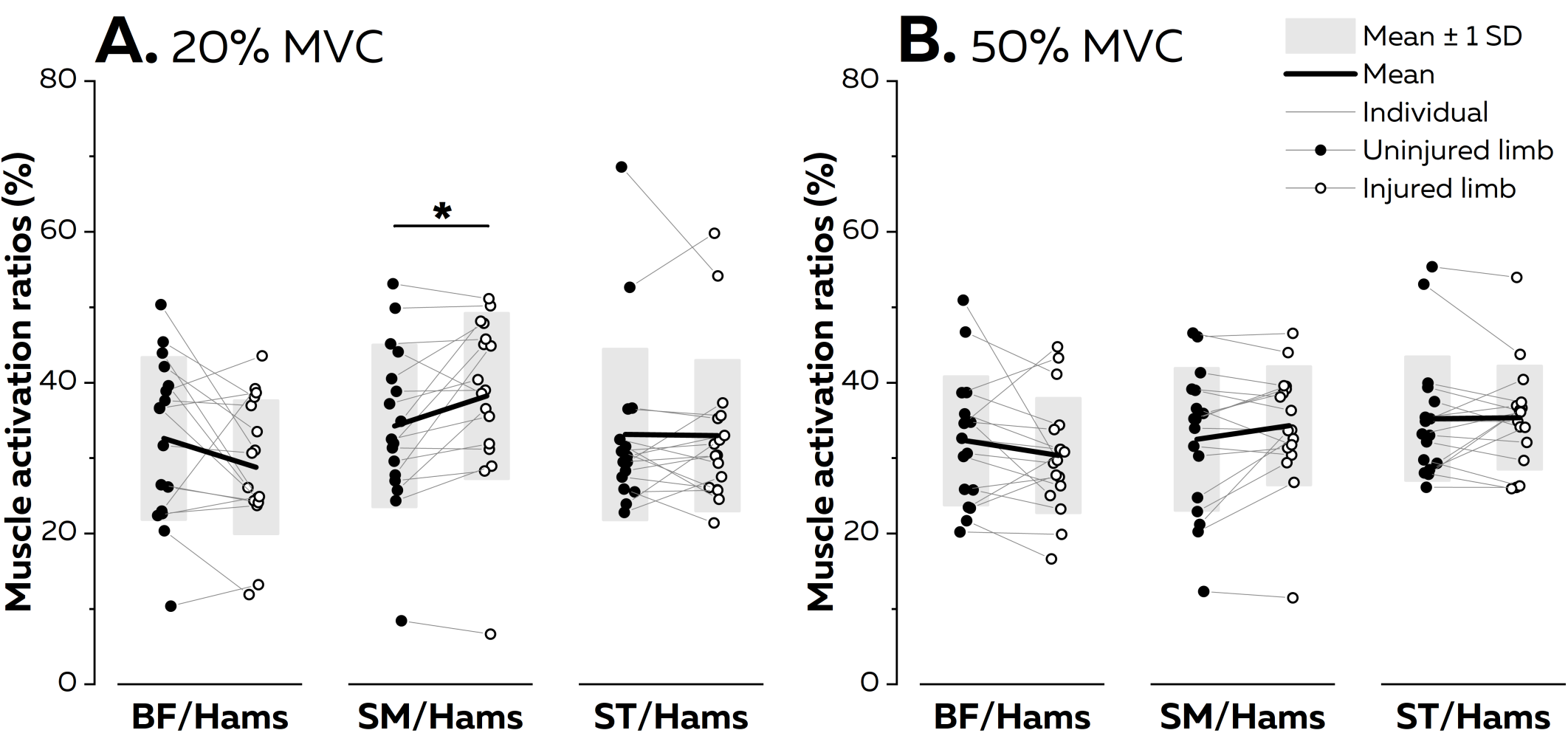


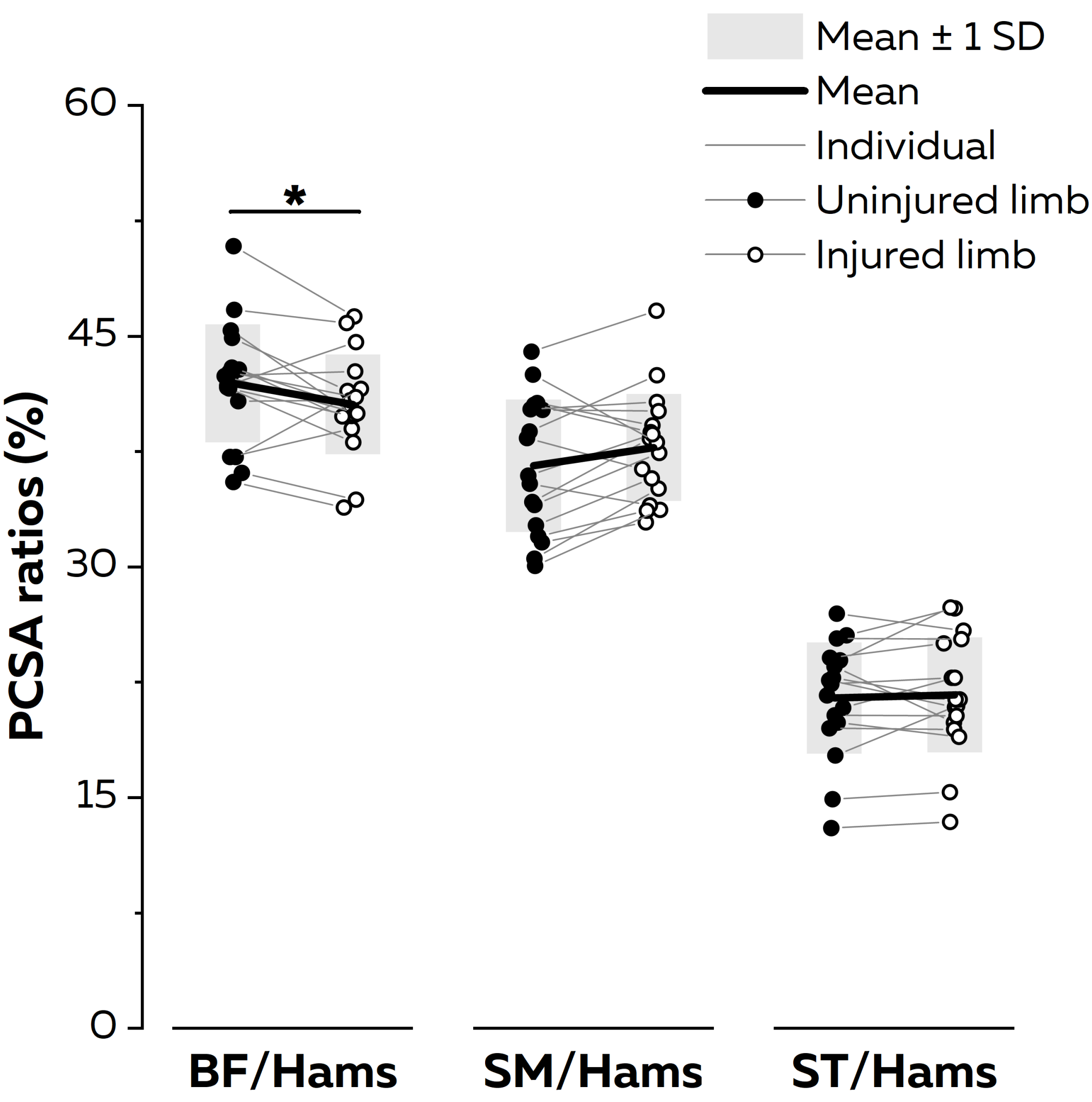



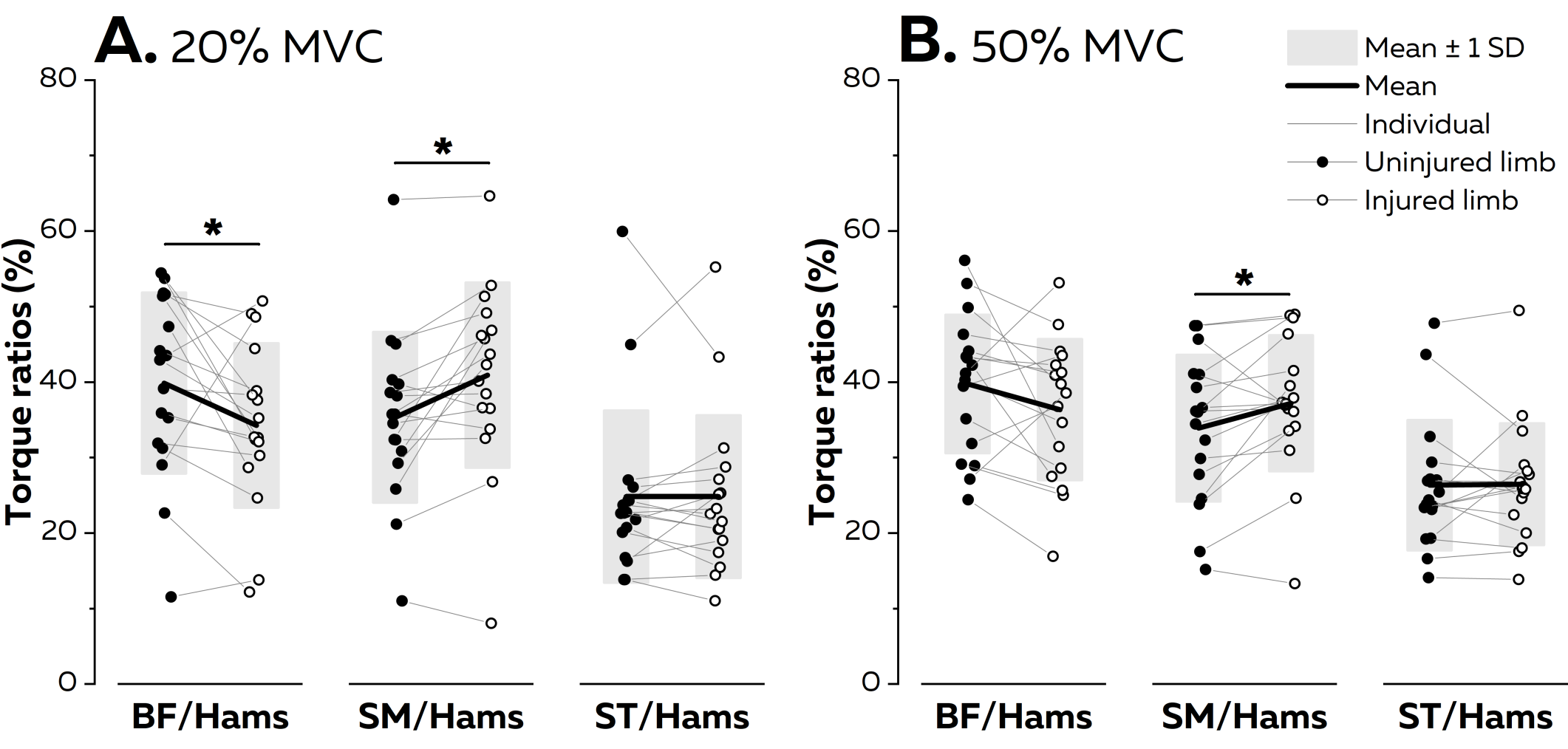